International Journal of Pure and Applied Mathematics

Volume 106 No. 2 2016, 677-687

ISSN: $1311-8080$ (printed version); ISSN: 1314-3395 (on-line version)

url: http://www.ijpam.eu

doi: 10.12732/ijpam.v106i2.29

\title{
NOTE ON THE DIMENSION OF THE MYCIELSKIAN OF A GRAPH
}

\author{
Peter Hudák ${ }^{1}$, Tomáš Madaras ${ }^{2}$, Pavol Široczki ${ }^{3}$ \\ ${ }_{1,2,3}$ Institute of Mathematics \\ Faculty of Science \\ P.J. Šafárik University \\ Jesenná 5, 04001 Košice, SLOVAKIA
}

\begin{abstract}
The dimension of a graph $G$ is the smallest integer $k$ such that $G$ has a unitdistance representation in Euclidean space $\mathbb{R}^{k}$. In this note, we study the relation between the dimensions of a graph and its Mycielskian.
\end{abstract}

AMS Subject Classification: $05 \mathrm{C} 62$

Key Words: Mycielskian, unit-distance graph, dimension of a graph

\section{Introduction}

In this note we consider simple graphs which will be represented in an Euclidean space $\mathbb{R}^{k}$ so that each edge is drawn as a unit line segment and distinct vertices correspond to distinct points of $\mathbb{R}^{k}$ (that is, the representation is nondegenerate); such a representation always exists for particular $k$ (it is enough to take $k$ equal to the number of vertices of a graph). Given a graph $G$, the smallest $k$ with this property is the dimension of $G$ and is denoted by $\operatorname{dim}(G)$. The graphs of dimension at most 2 are called unit-distance graphs.

Unit-distance graphs are studied mainly in connection with the (Hadwiger) Nelson problem: to determine the smallest number of colors (the chromatic number of the plane) needed to color points of $\mathbb{R}^{2}$ in such a way that any

Received: December 19, 2015

Published: February 19, 2016

${ }^{\S}$ Correspondence author (c) 2016 Academic Publications, Ltd.

url: www.acadpubl.eu 
two points of distance one receive different colors. The exact value is known to be between 4 and 7 (for discussions pro and con particular values, see an excellent monograph of A. Soifer [3]). To exclude (perhaps the most often conjectured) value 4 , it would be enough to find, for an integer $k \geq 5$, a $k$ critical unit-distance graph (a graph $G$ is called $k$-critical, if $\chi(G)=k$ and every proper subgraph of $G$ is $(k-1)$-colorable); note that, although there are certain indications for supporting their existence (as mentioned in [3], Chapters 3 and 47.3), such graphs would be likely very large (see [2]). Hence, a more promising direction of the research in this area would concern the values of dimension of chromatically critical graphs. By [1], for a $k$-critical graph $G$, $\operatorname{dim}(G) \leq 2 k$ holds; on the other hand, this bound is, in many cases, far from being sharp. The aim of this note is to contribute to the study of unit-distance representations of critical graphs by exploring the dimension of graphs which are constructed by the Mycielski construction. Recall that, for a graph $G$ with $V(G)=\left\{v_{i}: i \in[1, n]\right\}$ (for $i, j \in \mathbb{N},[i, j]$ denotes the set $\{k \in \mathbb{N}: i \leq k \leq j\}$ ), its Mycielskian $M(G)$ is obtained in the following way: for each $i \in[1, n]$, take a new vertex $w_{i}$ and add new edges $v_{i} w_{j}, v_{j} w_{i}$ whenever $v_{i} v_{j} \in E(G)$, $i, j \in[1, n]$; in addition, add a new vertex $u$ and add new edges $u w_{i}$ for all $i \in[1, n]$. If $G$ is $k$-critical, then $M(G)$ is $(k+1)$-critical (see [4], p. 206), hence $\operatorname{dim}(M(G)) \leq 2(k+1)$; however, apart from the trivial observation that $\operatorname{dim}(M(G)) \geq \operatorname{dim}(G)$, the relation between dimensions of $G$ and $M(G)$ is unclear. Unit-distance Mycielskians are briefly mentioned in [3], Chapter 15.1: for any graph containing a vertex of degree at least 3, its Mycielskian contains a copy of $K_{2,3}$, hence, it is not a unit-distance graph (as it is well known that $K_{2,3}$ is not a unit-distance graph; see [1]). Also, a unit-distance drawing of $M\left(C_{10}\right)$ is presented in this book and it is mentioned (but, without a proof) that Mycielskians of odd cycles do not have a unit-distance drawing in $\mathbb{R}^{2}$. We discuss these topics further in the next section, showing that the dimension of Mycielskians of cycles is at most 3 (the bound being attained for cycle of length at most 5) and conjecturing that Mycielskians of unions of paths have dimension 2. We also prove that all trees have a unit-distance drawing in $\mathbb{R}^{3}$ and that the dimension of the Mycielskian of $K_{n}$ is exactly $n$.

\section{The Results}

First, we discuss the case of graphs of dimension 1.

If $G \simeq 2 K_{1}$ then $M(G)$ consists of two isolated vertices and a graph $P_{3}$, thus obviously $\operatorname{dim}(M(G))=1$; further, $M\left(K_{2}\right) \simeq C_{5}$ has dimension 2 . Hence, 
assume that $G$ is of order $n \geq 3$. Then $\Delta(M(G))=n \geq 3$, thus, $\operatorname{dim}(M(G)) \geq$ 2.

Note that $\operatorname{dim}(G)=1$ implies that $G$ is a union of paths (of nonzero length) and isolated vertices. As isolated vertices of $G$ result in pendant vertices incident with an $n$-vertex of $M(G)$, we may assume that $G$ is a union of paths; then $M(G)$ is a union of blocks with the common cut-vertex of degree $n$.

The procedure for constructing a unit-distance representation of a single block of $M(G)$ corresponding to a single path $P=v_{1} v_{2} \ldots v_{k}$ starts by embedding 5-cycle $v_{1} v_{2} w_{1} u w_{2}$ : place $u$ arbitrarily, then place $w_{1}, w_{2}, v_{1}$ and $v_{2}$ in such a way that the edges have unit length and the distance between $u$ and $v_{1}$ as well as between $u$ and $v_{2}$ is smaller than 2. The vertices $u$ and $v_{2}$ determine the position of vertex $w_{3}$ being in unit distance from both of them (as their distance is smaller than 2 , there are two such points, one of them is $w_{1}$ ). In an analogous way, the position of vertex $v_{3}$ will be determined by vertices $v_{2}$ and $w_{2}$; because of the choice of their position, the distance between $u$ and $v_{3}$ will again be smaller than 2 . Thus, proceeding in general, the position of $v_{i}$ is uniquely determined by $v_{i-1}$ and $w_{i-1}$ and the position of $w_{i}$ is uniquely determined by $u$ and $v_{i-1}$.

If $G$ consists of several paths, we embed all the paths in the above described way and rotate each partial drawing of the Mycielskian of a single path by a small angle about $u$ to avoid possible identifications of vertices in the final drawing.

In principle, it is possible to express the position of each vertex as a pair of functions of two variables $\alpha$ and $\beta$ when initially setting $u=[0,0], w_{1}=$ $[0,1], w_{2}=[\cos \alpha, \sin \alpha], v_{2}=[1+\cos \beta, \sin \beta]$; note, however, that already the expressions for $v_{1}$ (being in unit distance from $v_{2}$ and $w_{2}$ ) are very complicated. Currently, we conjecture that there exists an initial assignment of the values of $\alpha$ and $\beta$ such that all the points $v_{i}, w_{i}, i \in[1, n]$ are distinct, but this is so far supported only indirectly by simulations in Wolfram Mathematica computer algebra system - we have developed a procedure that constructs, for given parameters $\alpha, \beta$ and a positive integer $n$, a unit-distance drawing of the Mycielskian of $n$-vertex path in the way described above. In addition, using the dynamic visualization features of Wolfram Mathematica, it is possible to vary the values of parameters and retrieve the resulting drawing online. Hence, we leave as open the following

Conjecture 1. If $\operatorname{dim}(G)=1, G ¥ 2 K_{1}$ then $\operatorname{dim}(M(G))=2$.

Next, we turn our attention to cycles, starting with the following observations: 
Observation 2. $\operatorname{dim}\left(M\left(C_{3}\right)\right)=3$.

Proof. The only possible way to draw $M\left(C_{3}\right)$ without vertex $u$ in $\mathbb{R}^{2}$ in a unit-distance way is illustrated in the right picture of Figure 1; but, then it is not possible to place vertex $u$ to have all its incident edges of unit length. It is easy to see that $M\left(C_{3}\right)$ has a unit-distance drawing in $\mathbb{R}_{3}$ : the triangles $v_{1} v_{2} w_{3}$, $v_{2} v_{3} w_{1}$ and $v_{3} v_{1} w_{2}$ can be tilted along edges $v_{1} v_{2}, v_{2} v_{3}$ and $v_{3} v_{1}$ respectively, raising vertices $w_{3}, w_{2}$ and $w_{1}$ to the same height above the plane containing $v_{1} v_{2} v_{3}$; finally, $u$ is chosen above $w_{1}, w_{2}, w_{3}$ to be at unit-distance from them.
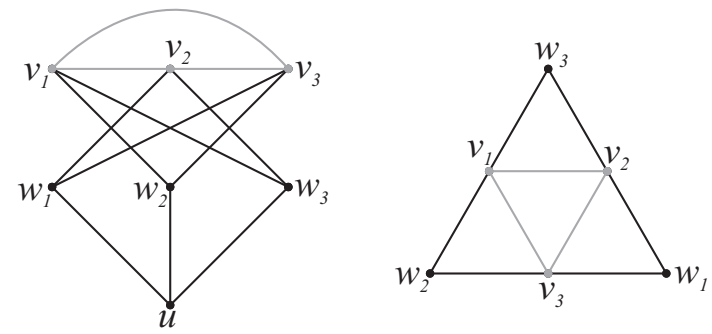

Figure 1: The graph $M\left(C_{3}\right)$

Observation 3. $\operatorname{dim}\left(M\left(C_{4}\right)\right)=3$.

Proof. Similarly to the previous case, it is easy to find a unit-distance representation of $M\left(C_{4}\right)$ in $\mathbb{R}^{3}$; on the other hand, $M\left(C_{4}\right)$ contains $K_{2,3}$ as a subgraph, hence it is not a unit-distance graph.

The case of $M\left(C_{5}\right)$ turns out to be more interesting. First, we deal with the dimension of the Wagner graph (that is, Möbius graph on 8 vertices):

Lemma 4. The Wagner graph has no unit-distance drawing in the Euclidean plane.

Proof. By contradiction, assume there exists a unit-distance drawing $D$ of the Wagner graph $W$ (see Figure 2). Note that $W$ contains a 4-cycle $C_{2}=$ $v_{3} v_{4} v_{6} v_{5}$ sharing two edges $v_{3} v_{4}, v_{5} v_{6}$ with two more 4-cycles: $C_{1}=v_{1} v_{2} v_{4} v_{3}$ and $C_{3}=v_{5} v_{6} v_{8} v_{7}$. Each such cycle must be drawn as a rhombus, thus edges $v_{1} v_{2}, v_{3} v_{4}, v_{5} v_{6}$ and $v_{7} v_{8}$ have to be parallel. Without loss of generality, let $v_{1}=[0,0]$ and $v_{2}=[1,0]$. Denote the measure of $\angle v_{2} v_{1} v_{3}$ as $\alpha$, the measure of $\angle v_{4} v_{3} v_{5}$ as $\beta$ and the measure of $\angle v_{6} v_{5} v_{7}$ as $\gamma$. Then

$$
v_{7}=[\cos \alpha+\cos \beta+\cos \gamma, \sin \alpha+\sin \beta+\sin \gamma],
$$




$$
v_{8}=[\cos \alpha+\cos \beta+\cos \gamma+1, \sin \alpha+\sin \beta+\sin \gamma] .
$$

In $D$, the edges $v_{1} v_{8}$ and $v_{2} v_{7}$ have to be of unit lengths, thus

$$
\begin{aligned}
& d\left(v_{1}, v_{8}\right)=(\cos \alpha+\cos \beta+\cos \gamma+1)^{2}+(\sin \alpha+\sin \beta+\sin \gamma)^{2}=1^{2}, \\
& d\left(v_{2}, v_{7}\right)=(\cos \alpha+\cos \beta+\cos \gamma-1)^{2}+(\sin \alpha+\sin \beta+\sin \gamma)^{2}=1^{2} .
\end{aligned}
$$

This implies $(\cos \alpha+\cos \beta+\cos \gamma+1)^{2}=(\cos \alpha+\cos \beta+\cos \gamma-1)^{2}$ which yields $\cos \alpha+\cos \beta+\cos \gamma=0$. Hence $v_{7}=[0, k]$ and as the length of $v_{2} v_{7}$ is 1 , this gives $d\left(v_{2}, v_{7}\right)=1^{2}+k^{2}=1^{2}$. Thus $k=0$ and $v_{7}=[0,0]$, a contradiction, because vertices $v_{1}$ and $v_{7}$ are identified in $D$.
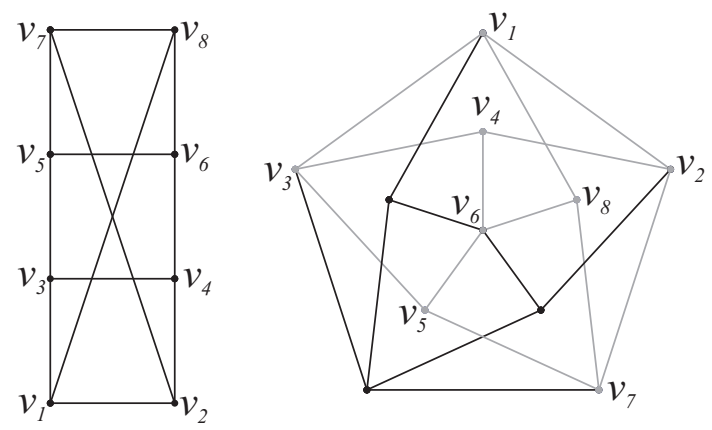

Figure 2: The Wagner graph and $M\left(C_{5}\right)$

Corollary 5. $\operatorname{dim}\left(M\left(C_{5}\right)\right)=3$.

Proof. The graph $M\left(C_{5}\right)$ contains the Wagner graph as a subgraph (see Figure 2), thus, its dimension is at least 3. The unit-distance embedding of $M\left(C_{5}\right)$ in $\mathbb{R}^{3}$ is illustrated by Figure 3 .

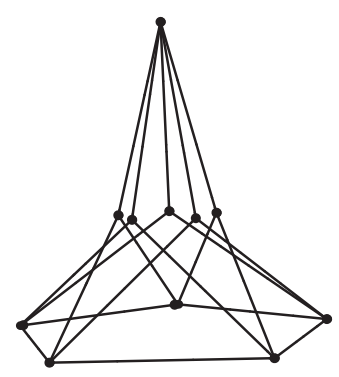

Figure 3: The unit-distance representation of $M\left(C_{5}\right)$ in $\mathbb{R}^{3}$ 
Theorem 6. For all $n \geq 7, \operatorname{dim}\left(M\left(C_{n}\right)\right) \leq 3$.

Proof. First we show that, for each $n \geq 7$, it is possible to draw the cycle $C_{n}=\left[v_{1} v_{2} \ldots v_{n}\right]$ in $\mathbb{R}^{2}$ in a unit-distance way such that all vertices lie on a geometric circle $C$ of radius $r_{n}<1$. In such a drawing, the edges of $C_{n}$ will then form chords of $C$, each of them having assigned the central angle of $C$ of the same measure $\varphi$ (we assume $\varphi \leq \pi$; otherwise we can take the complementary angle). We can express $\varphi=2 \arcsin \frac{1}{2 r_{n}}$ (as $r_{n}<1$ and $\varphi>\frac{\pi}{3}$ holds because $n \geq 7$ ) and there exists a positive integer $k$ such that $n \varphi=2 k \pi$. As the vertices of $C_{n}$ shall not be identified, $k$ and $n$ have to be coprime. Thus, we obtain $\frac{2 k \pi}{n}=2 \arcsin \frac{1}{2 r_{n}}$ which yields $r_{n}=\frac{1}{2 \sin \left(\frac{k \pi}{n}\right)}<1$. If $\left\lfloor\frac{n}{6}\right\rfloor+1 \leq k \leq\left\lceil\frac{n}{2}\right\rceil-1$ is satisfied, the previous inequality holds.

The Bertrand's postulate gives that if $\left\lfloor\frac{n}{4}\right\rfloor \geq 2$ (this holds for $n \geq 8$; for $n=7$ take for example $k:=2$ ), then there exists a prime number $p_{1}$ with $\left\lfloor\frac{n}{4}\right\rfloor<p_{1}<2\left\lfloor\frac{n}{4}\right\rfloor$. It is simple to verify that $p_{1}$ satisfies our conditions on $k$ hence, if $p_{1}$ and $n$ are coprime, then we can take $k=p_{1}$. On the other hand, $n$ and $p_{1}$ are not coprime only if $n=3 p_{1}$. In this case, by applying Bertrand's postulate again, we get that, for $\left\lfloor\frac{n}{6}\right\rfloor \geq 2$ (with $n \geq 12$; for $n=9$ take $k:=4$ ), there exists a prime number $p_{2}$ such that $\left\lfloor\frac{n}{6}\right\rfloor<p_{2}<2\left\lfloor\frac{n}{6}\right\rfloor$. Again, it is easy to verify that $\left\lfloor\frac{n}{6}\right\rfloor+1 \leq p_{2} \leq\left\lceil\frac{n}{2}\right\rceil-1$, thus, we can take $k=p_{2}$ if $n$ and $p_{2}$ are coprime. The only cases that $n$ and $p_{2}$ are not coprime are $n=4 p_{2}$ or $n=5 p_{2}$, which yield (together with $n=3 p_{1}$ ) $p_{2}=3$ and $n \in\{12,15\}$. For $n=12$ take $k:=5$. If $n=15$, we can take $k:=4$ getting the desired drawing of $C_{n}$ in $\mathbb{R}^{2}$ such that all points lie on a circle of a small radius.

Now, to obtain a unit-distance drawing of the Mycielskian of $C_{n}$ in $\mathbb{R}^{3}$, we need to suitably place vertices $w_{1}, \ldots, w_{n}$ and $u$. This can be done in the following way: construct the line $p_{1}$ being orthogonal to the plane $P$ of $C$ and passing through the center of the linear segment $v_{2} v_{n}$. Then place $w_{1}$ on $p_{1}$ to be at unit-distance from both $v_{2}$ and $v_{n}$. There are two suitable positions for $w_{1}$, choose either one. Repeating this construction for points $v_{i}$ and $v_{i+2}$ (indices are taken modulo $n$ ) and placing every new point $w_{i}, i \in[2, n]$ in the same subspace generated by $P$ as vertex $w_{1}$, we obtain all the points $w_{i}, i \in[1, n]$, lying on a small circle of radius less than 1 . The construction is completed by finding the position of $u$ being at unit-distance from all $w_{i}, i \in[1, n]$ which can be easily done.

We note, that similarly it is easy to show that $\operatorname{dim}\left(M\left(C_{6}\right)\right) \leq 3$, starting with a regular hexagon in the plane and placing the remaining vertices suitably.

Nothing is known about the difference $\operatorname{dim}(M(G))-\operatorname{dim}(G)$; we do not even know an example of a unit-distance graph whose Mycielskian has the dimension 
at least 4. However, an easy attempt to find it by establishing the existence of a large complete bipartite graph (which has dimension equal to 4) in Mycielskian fails due to the following

Theorem 7. If $M(G)$ contains $K_{3,3}$ as a subgraph, then $G$ contains $K_{2,3}$ as a subgraph.

Proof. Let $H$ be a copy of $K_{3,3}$ in $M(G)$ and assume $G$ does not contain $K_{2,3}$ as a subgraph. Assume first that $u \in V(H)$. Then $H$ has a bipartition $\left\{w_{1}, w_{2}, w_{3}\right\}$ (indices are chosen without loss of generality). Furthermore, $H$ must contain two vertices from $V(G)$ which are different from $v_{1}, v_{2}$ and $v_{3}$ (as the vertex $v_{i}$ is not incident with $w_{i}$ ); denote them $v_{4}$ and $v_{5}$. Then $v_{4}$ and $v_{5}$ are incident with $v_{1}, v_{2}$ and $v_{3}$, thus forming $K_{2,3}$ in $G$.

If $u \notin V(H)$ and $|V(H) \cap V(G)| \geq 5$, then $K_{2,3} \subseteq G$. Otherwise, if $u \notin V(H)$ and $|V(H) \cap V(G)| \leq 4$, this means that there exist (without loss of generality) vertices $w_{1}, w_{2} \in V(M(G)) \backslash(V(G) \cup\{u\})$ which are vertices of $H$; moreover, $w_{1}$ and $w_{2}$ belong to the same bipartition of $H$, as they are not adjacent in $M(G)$. It is also obvious, that the other color class only contains vertices from $V(G)$ (because of the character of the Mycielski construction), say $v_{3}, v_{4}$ and $v_{5}$. As $w_{1}$ and $w_{2}$ are connected by edges to vertices $v_{3}, v_{4}$ and $v_{5}$, the same applies to vertices $v_{1}$ and $v_{2}$ in $G$. Note that the vertices $v_{i}, i \in[1,5]$ are distinct. But this gives that $v_{1}, v_{2}, v_{3}, v_{4}$ and $v_{5}$ form a copy of $K_{2,3}$ as a subgraph in $G$ (with bipartition $\left\{v_{1}, v_{2}\right\}$ and $\left\{v_{3}, v_{4}, v_{5}\right\}$ ).

Therefore, it is necessary to use an approach based on looking for other subgraphs - possibly, triangle-free graphs - of dimension 4 in Mycielskians, but such graphs are, so far, only little explored.

Theorem 8. Let $T$ be a tree. Then $\operatorname{dim} M(T) \leq 3$ and there exists a unitdistance drawing $D$ of graph $M(T)$ in $\mathbb{R}^{3}$ with $\max \{d(x, y): x, y \in V(D)\}<2$.

Proof. By induction on number of vertices. It is easy to see that Mycielskians of trees on at most two vertices have the desired properties, thus, the base case holds.

Induction step: We assume that the theorem holds for every tree on at most $n \geq 2$ vertices. Let $T$ be a tree on $n+1$ vertices. As $T$ has at least 3 vertices, it contains at least two vertices of degree 1 ; denote one of them as $v_{1}$. Let $v_{2}$ be the neighbour of $v_{1}$ and let $T_{1}=T-v_{1}$. The graph $T_{1}$ satisfies the induction hypothesis, thus $\operatorname{dim} M\left(T_{1}\right) \leq 3$ and there exists a drawing $D_{1}$ of $M\left(T_{1}\right)$ such that $\max \left\{d(x, y): x, y \in V\left(D_{1}\right)\right\}<2$. The second part can be interpreted as follows: there exists a closed unit ball $\mathcal{B}_{1}$ in $\mathbb{R}^{3}$ such that $D_{1} \subset$ 
$\mathcal{B}_{1}$. Further, $V(M(T)) \backslash V\left(M\left(T_{1}\right)\right)=\left\{v_{1}, w_{1}\right\}$ and $E(M(T)) \backslash E\left(M\left(T_{1}\right)\right)=$ $\left\{v_{1} v_{2}, v_{1} w_{2}, v_{2} w_{1}, u w_{1}\right\}$; therefore, to obtain a suitable unit-distance drawing $D$ of $M(T)$, we need to add two vertices and four edges to $D_{1}$. The position of vertex $w_{1}$ is determined by positions of $v_{2}$ and $u$. Set $\mathcal{C}_{1}=\left\{x \in \mathbb{R}^{3}\right.$ : $\left.d(u, x)=1 \wedge d\left(v_{2}, x\right)=1\right\}$; then $\mathcal{C}_{2}:=\mathcal{B}_{1} \cap \mathcal{C}_{1}$ is a subset of a circle in $\mathbb{R}^{3}$. In a constructed drawing, every edge is represented by a line segment, hence, the intersection of any edge with $\mathcal{C}_{2}$ consists of at most two points. Furthermore, as only finitely many vertices of $D_{1}$ can lie on $\mathcal{C}_{2}$ and the set $D^{\prime}=\left\{x \in \mathcal{C}_{2}:\left(x v_{2} \cup x u\right) \cap V\left(D_{1}\right) \neq \emptyset\right\}$ is finite, there are only finitely many points of $\mathcal{C}_{2}$ which are not good for placing vertex $w_{1}$. Hence, we can place $w_{1}$ in such a way that the edges $u w_{1}$ and $v_{2} w_{1}$ are of unit length. Similarly, the position for vertex $v_{1}$ is determined by vertices $w_{2}$ and $v_{2}$. Repeating an analogous argumentation as in the previous case we obtain a suitable position for vertex $v_{1}$ and edges $v_{1} v_{2}$ and $v_{1} w_{2}$, thus obtaining a unit-distance drawing $D$ of $M(T)$ which is contained in $\mathcal{B}_{1}$.

Theorem 9. For all $n \in \mathbb{N}, \operatorname{dim}\left(M\left(K_{n}\right)\right)=n$.

Proof. First we show that, for any $n \in \mathbb{N}$, the graph $M\left(K_{n}\right)$ has a unitdistance drawing in $\mathbb{R}^{n}$. Later we will show that a unit-distance drawing of such a graph in $\mathbb{R}^{n-1}$ does not exist.

For fixed $n$, let us consider the graph $K_{n}$ with the vertex set $\left\{v_{i}: i \in\right.$ $[1, n]\}$ and let $H:=M\left(K_{n}\right)$ have the vertex set $\left\{u, v_{i}, w_{i}: i \in[1, n]\right\}$, as described, together with its edge set, in the introduction. Now, identify the vertex $v_{i}$ with a point of $\mathbb{R}^{n}$ with the $i$-th coordinate being $\frac{\sqrt{2}}{2}$ and all remaining coordinates being 0 . Similarly, identify the vertex $w_{i}$ with a point of $\mathbb{R}^{n}$ where the $i$-th coordinate is $-\frac{\sqrt{2}}{2}$ and the other coordinates are 0 . To complete the construction, identify the vertex $u$ with a point of $\mathbb{R}^{n}$ where all coordinates are equal to a real number $x$; we will show that it is possible to find a suitable value for $x$ so that the obtained drawing is a unit-distance drawing.

For any $i, j \in[1, n]$ it follows that if the vertices $v_{i}$ and $w_{j}$ form an edge, then their distance is 1 . Similarly, any edge $v_{i} v_{j}$ can be seen to be of unit length. Consider now the edge $w_{i} u$ for $i \in[1, n]$. Its length can be expressed as $\sqrt{(n-1) \cdot x^{2}+\left(x+\frac{\sqrt{2}}{2}\right)^{2}}$ which has to be equal to 1 . This is equivalent with the equation $n x^{2}+x \sqrt{2}-\frac{1}{2}=0$ which clearly has two real solutions, each of them being suitable for obtaining a unit-distance drawing of $H$ in $\mathbb{R}^{n}$.

It remains to show that there is no unit-distance drawing of $H$ in $\mathbb{R}^{n-1}$. It is easy to see that the statement holds for $n \leq 2$. The proof is completed by showing the following statement: 
Lemma 10. For each $n \geq 3$, the unit-distance drawing $D_{n}$ of $K_{n}$ in $\mathbb{R}^{n-1}$ is unique up to rotations and translations. Furthermore, for a fixed $D_{n}$, the positions of vertices $w_{i}, i \in[1, n]$, in a possible unit-distance drawing of $M\left(K_{n}\right)$ are uniquely determined and there exists exactly one point $S_{n} \in \mathbb{R}^{n-1}$ such that there is a real number $c_{n}$ with $d\left(w_{i}, S_{n}\right)=c_{n}$ for each $i \in[1, n]$; additionally, $c_{n}>1$ holds.

\section{Proof. By induction on $n$.}

Base step: for $n=3$ the only unit-distance drawing of $K_{3}$ in $\mathbb{R}^{2}$ is an equilateral triangle, so, without loss of generality, we can fix $D_{3}$ as a drawing, where we identify the vertex $v_{1}$ with the point $[0,0], v_{2}$ with the point $[1,0]$ and $v_{3}$ with the point $\left[\frac{1}{2}, \frac{\sqrt{3}}{2}\right]$. The position of vertex $w_{1}$ is uniquely determined by the positions of $v_{2}$ and $v_{3}$ and the fact it can not be mapped to the same point as $v_{1}$; it is equal to $\left[\frac{3}{2}, \frac{\sqrt{3}}{2}\right]$. Similarly, the position of $w_{2}$ is necessarily $\left[-\frac{1}{2}, \frac{\sqrt{3}}{2}\right]$ and $w_{3}$ is at point $\left[\frac{1}{2},-\frac{\sqrt{3}}{2}\right]$. It is a simple geometric observation that $w_{1}, w_{2}$ and $w_{3}$ form an equilateral triangle, thus, the only point $S_{3}$ in equal distance from them is the center $\left[\frac{1}{2}, \frac{\sqrt{3}}{6}\right]$ of this triangle. For $i \in[1,3], c_{3}:=d\left(w_{i}, S_{3}\right)=\frac{2 \sqrt{3}}{3}>1$.

Induction step: Assume the statement is true for every $k \leq n-1$. For a unitdistance drawing of $K_{n}$ in $\mathbb{R}^{n-1}$, consider first its subgraph $\widetilde{K}=K_{n}-v_{n} \cong K_{n-1}$ with vertices $v_{i}, i \in[1, n-1]$. Due to the induction hypothesis, $\widetilde{K}$ has a unique unit-distance drawing $\widetilde{D}$ in $\mathbb{R}^{n-2}$. Translate and rotate $\widetilde{D}$ in such a way that the all coordinates of the point $S_{n-1}$ are 0 . Now $S_{n-1}$ is the center of the convex $(n-1)$-simplex formed by the vertices of $\widetilde{D}$ and, therefore, it is the only point of $\mathbb{R}^{n-2}$ in the same distance from all vertices $v_{i}, i \in[1, n-1]$. Set $d_{1}:=d\left(v_{1}, S_{n-1}\right)$ and assume that $d_{1}>1$. All the vertices of $\widetilde{D}$ have to lie on the $(n-2)$-dimensional sphere $\mathcal{S}_{1}$ with center $S_{n-1}$ and radius $d_{1}$. Consider a second sphere $\mathcal{S}_{2}$ with center $v_{1}$ and radius 1 . For each $i \in[2, n-1], d\left(v_{1}, v_{i}\right)=1$, thus each $v_{i}$ lies on $\mathcal{S}_{2}$ and the convex hull of these vertices (the simplex itself) is a subset of the closed ball $\mathcal{B}_{2}$ with boundary $\mathcal{S}_{2}$. However, as $d_{1}>1, S_{n-1}$ lies outside of $\mathcal{B}_{2}$, a contradiction. If $d_{1}=1$, we construct $\mathcal{S}_{1}$ and $\mathcal{S}_{2}$ as in the previous case. The convex hull $M$ of the set $\left\{v_{i}, i \in[1, n-1]\right\}$ is again a subset of $\mathcal{B}_{2}$ and, as $S_{n-1}$ lies on the boundary of $\mathcal{B}_{2}$, it belongs to $M$ if and only if it is one of the exterior points $v_{i}, i \in[1, n-1]$, which contradicts the assumption that $d\left(S_{n-1}, v_{i}\right)=d_{1}=1$ for each $i \in[1, n-1]$.

Now we can assume that $d_{1}<1$. Note that we need to place the vertex $v_{n}$ at a point $P$ such that all the distances $d\left(P, v_{i}\right), i \in[1, n-1]$ are equal. Since $P$ lies on the line passing through $S_{n-1}$ and perpendicular to the $(n-2)$-dimensional subspace spanned by points $v_{i}, i \in[1, n-1]$, the first $n-2$ coordinates of $P$ are equal to 0 . As $d_{1}<1$, the last coordinate of $P$ can be chosen in such a way that 
$d\left(v_{n}, v_{i}\right)=1$ for each $i \in[1, n-1]$. Actually, there are two such points (in one case, take the $(n-1)$-th coordinate to be positive, in the other negative with the same value), but both drawings are symmetric under a symmetry through the $(n-2)$-dimensional subspace spanned by the points of $\widetilde{D}$ we considered earlier.

The position of the vertex $w_{n}$ is then determined uniquely - it is placed as the counterpart of point $v_{n}$ in the afore mentioned symmetry. Vertices $w_{i}$, $i \in[1, n-1]$ are then placed similarly as $w_{n}$ (each of them has to be the counterpart of the vertex $v_{i}$ under a symmetry through the $(n-2)$-dimensional subspace spanned by the set $\left.\left\{v_{j}: j \in[1, n], j \neq i\right\}\right)$.

It is a simple observation that the point $S_{n}$ lies on the line passing through the points $v_{n}$ and $S_{n-1}$ (as all the points in equal distance from points $v_{i}$, $i \in[1, n-1]$ must lie on this line; these are also the points of equal distance from $\left.w_{i}, i \in[1, n-1]\right)$. Then, without loss of generality, we can set $c_{n}=d\left(w_{1}, S_{n}\right) \geq$ $d\left(w_{1}, S_{n-1}\right)=c_{n-1}>1$ (as the point $S_{n-1}$ is the orthogonal projection of $S_{n}$ onto the $(n-2)$-dimensional subspace spanned by $\left.v_{i}, i \in[1, n-1]\right)$, thus completing the proof of our statement.

Corollary 11. For arbitrary graph $G$ : $\operatorname{dim}(M(G)) \leq|V(G)|$.

Note that this bound improves the upper bound $2 \chi(M(G))$ for each graph $G$ with $\chi(G) \geq \frac{|V(G)|}{2}-1$.

\section{Acknowledgments}

This work was supported by the Slovak Science and Technology Assistance Agency under the contract APVV-0023-10, Slovak VEGA grant 1/0652/12, VVGS-2013-109 and VVGS-PF-2014-447. 


\section{References}

[1] P. Erdős, F. Harary, W.T. Tutte, On the dimension of a graph, Mathematika, 12, No. 2 (1965), 118-122, doi: 10.1112/s0025579300005222.

[2] D. Pritikin, All unit-distance graphs of order 6197 are 6-colorable, Journal of Combinatorial Theory, Series B, 73, No. 2 (1998), 159-163, doi: 10.1006/jctb.1998.1819.

[3] A. Soifer, The Mathematical Coloring Book, Springer, USA (2009), doi: 10.1007/978-0387-74642-5.

[4] D. West, Introduction to Graph Theory, Prentice Hall, USA (2001). 
